\title{
On the Capacity of Ad Hoc Wireless Networks Under General Node Mobility
}

\author{
Michele Garetto, Paolo Giaccone, Emilio Leonardi*
}

\begin{abstract}
We revisit the problem of characterizing the capacity of an ad hoc wireless network with $n$ mobile nodes. Grossglauser and Tse (2001) showed that, by exploiting user mobility, it is possible to maintain a constant per-node throughput as the number of nodes grows. Their scheme allows to overcome the throughput decay (at least as $1 / \sqrt{n}$ ) that affects networks with static nodes, which was first pointed out by Gupta and Kumar (2000). Subsequent works have analyzed the delaycapacity trade-off that arises in mobile networks under various mobility models. Almost invariably, however, available asymptotic results strongly rely on the assumption that nodes are identical, and move according to some ergodic process that is equally likely to visit any portion of the network area. In this paper, we relax such 'homogeneous mixing' assumption on the node mobility process, and analyze the network capacity in the more realistic case in which nodes are heterogeneous, and the motion of a node does not necessarily cover uniformly the entire space. We propose a general framework to characterize the capacity of networks with arbitrary mobility patterns, considering both the case of finite number of nodes (also with the support of experimental traces), as well as asymptotic results when the number of nodes grows to infinity.
\end{abstract}

\section{INTRODUCTION AND PREVIOUS WORK}

In recent years a significant effort has been devoted by the research community to study the asymptotic performance of ad hoc wireless networks when the number of users increases. Gupta and Kumar [1] consider a model in which $n$ static nodes randomly placed in a disk of unit area, establish $n$ source-destination (S-D) communications. They obtain the disheartening result that the throughput available to each S-D pair decreases at least as $1 / \sqrt{n}$, even allowing optimal scheduling and node placement. Grossglauser and Tse [2] consider a similar scenario in which the nodes are mobile, and show that, in contrast to the fixed node case, the throughput per S-D pair can be kept constant while increasing $n$. This nice property was established under the assumption that the trajectories of the nodes are independent, and results for each node into a uniform stationary distribution over the disk of unit area. This mobility pattern is actually a generous one, since it allows each node to equally come in contact with any other node in the network.

Indeed, in real environments, the contact times between the nodes can be highly diverse, as recently pointed out in [3]. Actually, an individual node usually spends most of the time just on a small portion of the entire network area, and rarely goes outside its region of habit. Motivated by this observation, in [4] the authors considered a restricted

* M. Garetto is with Dipartimento di Informatica, Università di Torino, Italy; P. Giaccone and E. Leonardi are with Dipartimento di Elettronica, Politecnico di Torino, Italy. This work was supported by the Italian Ministry of University and Research through CARTOON PRIN-project. mobility model, where each node independently moves along a randomly chosen great circle on the sphere of unit surface. Quite surprisingly, even under this one-dimensional mobility pattern a constant throughput per S-D pair can be sustained.

Since then, the attention of researchers has been mostly attracted by capacity-delay trade-offs [5], [6], [7], [8]. Various mobility models have been considered, such as the simple reshuffling model [5], Brownian motion [6], different variants of random walks and random way-point [7], [8]. Almost invariably, in all these studies nodes are assumed to be identical and independently moving, while their trajectories 'fill the space over time'. One exception is [9], where the authors study throughput-delay scaling laws for the same one-dimensional mobility pattern considered in [4]. Another study of capacitydelay trade-off under restricted mobility appears in [10]: here the network of unit area is partitioned into square cells, and nodes are restricted to move within one randomly chosen cell; the authors consider two cases in which the cell area either scales as $(\log n) / n$ or remains constant, obtaining results close to Gupta-Kumar and Grossglauser-Tse, respectively.

Apart from the above examples of restricted mobility over great circles or cells, the general question about how anisotropic mobility patterns affect the network capacity has been left behind unanswered. The goal of this paper is to fill the existing gap in the analysis of the throughput capacity of ad hoc wireless networks. We address the general case of heterogeneous nodes with arbitrary mobility patterns. Clearly, there exists an extraordinary huge space of different mobility patterns in between the extreme cases of static nodes (GuptaKumar) and complete homogeneous mixing (GrossglauserTse). Therefore it is quite interesting to investigate how the network capacity can vary across such a huge space. In this work we are not concerned with delay, but only on throughput. Thus practical implications of our work mainly fit in the context of delay tolerant networks [11].

We remark that we are not the first to consider the capacity of ad hoc wireless networks with heterogeneous nodes. In the particular case of static nodes, several works have already appeared that generalize the results of Gupta-Kumar. The nice deterministic approach proposed in [12] allows to analyze in a simple way non-uniform spatial distribution such as straight lines, or highly dense neighborhoods. The work in [13] investigates the network capacity resulting from asymmetric traffic patterns. In [14] the authors analyze arbitrary node placement and interference constraints using spectral techniques. Several papers have also considered the case of hybrid networks, where an overlay of wired base stations is added to the ad hoc network [15], [16], [17], [18], with the potential of dramatically improving the available per-node throughput. 
To the best of our knowledge, we are the first to consider the general case of heterogeneous mobile nodes. More specifically, we provide the following contributions: i) we formulate the general case as a joint scheduling and routing problem, defining an abstract framework within which the performance analysis of mobile ad hoc networks can be carried out; ii) we precisely characterize the capacity region of a network with finite number of nodes, pointing out several structural properties of the system; iii) we apply the framework to the analysis of a real network, using publicly available traces; iv) we extend the analysis to the asymptotic regime, first establishing some results of general validity, and then considering a few significant examples of anisotropic distributions of the nodes over the area.

\section{NETWORKS WITH FINITE NUMBER OF NODES}

\section{A. System assumptions and notation}

We consider a mobile ad hoc network composed of $n$ nodes moving according to a general mobility model inside a bidimensional, compact and convex region $\mathcal{A}$ of area $|\mathcal{A}|$. Let $X_{i}(t)$ denote the position of node $i$ at time $t$ and $\mathbf{X}(t)=\left(X_{1}(t), X_{2}(t) \ldots X_{n}(t)\right)$ the vector of nodes' positions; we define with $d_{i j}(t)$ the euclidean distance between mobile $i$ and mobile $j$ at time $t$, i.e., $d_{i j}(t)=\left\|X_{i}(t)-X_{j}(t)\right\|_{2}$.

We assume the node mobility process to be stationary and ergodic; i.e., given any $m$-uple $\left(B_{1}, B_{2}, B_{3} \ldots B_{m}\right)$ of Lebesgue measurable subsets of $A$, it results:

$$
\lim _{t \rightarrow \infty} \frac{1}{t} \int_{0}^{t} \mathbb{I}_{\left(\cap_{i} X_{i}(\tau) \in B_{i}\right)} d \tau=E\left[\mathbb{I}_{\left(\cap_{i} X_{i}(t) \in B_{i}\right)}\right] \quad \text { w.p. } 1
$$

where II represents the logical indicator function.

Node $s$ generates traffic for destination $d$ according to a stationary and ergodic process with average traffic rate $\lambda_{s d}$ bit $/ \mathrm{s}^{1}$. We denote with $\boldsymbol{\Lambda}=\left[\lambda_{s d}\right]$ the corresponding $n \times n$ traffic matrix.

We assume that interference between simultaneous transmissions is described by the well known protocol interference model [1]. However, most of the results obtained in this paper can be extended to the physical interference model [1] too ${ }^{2}$. According to the protocol interference model, transmission from node $i$ to node $j$ at time $t$ at rate $r$ is successful only if, for any other simultaneously transmitting node $k$, it holds:

$$
d_{k j}(t)>(1+\Delta) d_{i j}(t)
$$

for some guard factor $\Delta>0$. Note that according to this interference model: (i) no node can be either origin or destination of multiple simultaneous transmissions, (ii) a node cannot be simultaneously origin and destination of transmissions.

We denote with $Q$ the set of all possible transmissionreceiver pairs $(i, j)$ (by construction it must be $i \neq j$ ). Subsets $\pi$ of $Q$ in which nodes appear at most once (either as transmitter or receiver), represent possible transmission

\footnotetext{
${ }^{1}$ Defined with $\hat{\lambda}(t, \tau)$ the amount of data generated by a source within the interval $[t, \tau)$, the traffic is said stationary and ergodic with average rate $\lambda$ iff: $E[\hat{\lambda}(t, t+1)]=\lambda$ for any $t>0$ and $\lim _{t \rightarrow \infty} \hat{\lambda}(0, t) / t=\lambda$ w.p. 1 .

${ }^{2}$ Note that the protocol interference model has been proved to be equivalent to the physical interference model when each user employs the same power [1].
}

configurations, i.e., sets of transmission-receiver pairs $(i, j)$, which may be simultaneously enabled to communicate at time $t$. We denote with $\Pi$ the set of all the possible transmission configurations and with $A(t) \subseteq \Pi$ the set of all noninterfering (hence, implementable) transmission configurations at time $t$. The protocol interference model (more in general, any interference model) induces a correspondence between the vector of instantaneous nodes positions $\mathbf{X}(t)$ and the set of non-interfering transmission configurations $A(t)$; we formalize this concept introducing function $\mathcal{I}$ mapping vectors of nodes positions into sets of non-interfering transmission configurations: $\mathcal{I}(\mathbf{X}(t))=A(t)$.

Given any set $A$ of implementable transmission configurations, we denote with $\mathcal{I}^{-1}(A)$ the set of node positions $\mathbf{X}$ to which $A$ corresponds through mapping $\mathcal{I}$, i.e., $\mathcal{I}^{-1}(A)=$ $\{\mathbf{X}: \mathcal{I}(\mathbf{X})=A\} .{ }^{3}$ For any $A$, we can univocally determine the probability that $A(t)=A$, i.e. the probability that configurations in $A$ are the only implementable at time $t$ :

$P(A)=E\left[\mathbb{I}_{X(t) \in \mathcal{I}^{-1}(A)}\right]=\lim _{t \rightarrow \infty} \frac{1}{t} \int_{0}^{t} \mathbb{I}_{X(\tau) \in \mathcal{I}^{-1}(A)} d \tau$ w.p.1

Note that the above probability depends only on the joint stationary distribution of the node mobility process.

At last, we denote with $\left\{T_{n}\right\}$ the sequence of random instants at which the set of implementable transmission configurations changes; i.e., $\lim _{t \uparrow T_{n}} A(t) \neq A\left(T_{n}\right)$.

\section{B. Scheduling policy}

The scheduling policy $S$ dynamically selects an implementable transmission configuration $\pi^{S}(t)$ belonging to $A(t)=\mathcal{I}(\mathbf{X}(t))$. In this paper we restrict our investigation to stationary and ergodic scheduling policies: i.e. those policies for which:

$$
E\left[\mathbb{I}_{(i, j) \in \pi^{S}(t)}\right]=\lim _{t \rightarrow \infty} \frac{1}{t} \int_{0}^{t} \mathbb{I}_{(i, j) \in \pi^{S}(\tau)} d \tau \quad \text { w.p.1 }
$$

In general the selection of $\pi^{S}(t)$ may be influenced by several dynamical parameters, including instantaneous queues lengths, age of stored information at nodes, etc. Particularly relevant are those scheduling policies driven only by $\mathbf{X}(t)$. In this paper we call stateless and memoryless such scheduling policies.

We also introduce the class of simple scheduling policies $\hat{S}$, which is a strict subclass of the stateless and memoryless scheduling policies characterized as follows. At each transition time $T_{n}$ a transmission configuration $\pi \in A\left(T_{n}\right)$ is selected according to a stationary and memoryless (possibly random) rule; the selected transmission configuration is then kept constant in the whole interval $\left[T_{n}, T_{n+1}\right)$. Simple scheduling policies are fully specified by the conditional probabilities $p_{\hat{S}}(\pi, A)$ that the transmission configurations $\pi \in A$ are selected at time $T_{n}$, given that $X\left(T_{n}\right) \in \mathcal{I}^{-1}(A)$ :

$$
\begin{aligned}
p_{\hat{S}}(\pi, A)=\operatorname{Pr}\left\{\pi^{\hat{S}}\left(T_{n}\right)=\pi \mid X\left(T_{n}\right) \in \mathcal{I}^{-1}(A)\right\} & \\
& \forall A \text { and } \pi \in A
\end{aligned}
$$

According to scheduling policy $S$, a communication link is established between nodes $i$ and $j$ whose average capacity expressed in bit/s is:

$$
\mu_{i j}^{S}=r E\left[\mathbb{I}_{(i, j) \in \pi^{S}(t)}\right]=\lim _{t \rightarrow \infty} \frac{r}{t} \int_{0}^{t} \mathbb{I}_{(i, j) \in \pi^{S}(\tau)} d \tau \quad \text { w.p.1 }
$$

${ }^{3}$ It can be verified that, for any $A \in \Pi, \mathcal{I}^{-1}(A)$ is a convex set of $\mathbb{R}^{2 n}$. 
which, in case of simple scheduling policies, can be rewritten as: $\mu_{i j}^{\hat{S}}=r \sum_{A \in \Pi} \sum_{\pi \in A} \mathbb{I}_{(i, j) \in \pi} p_{\hat{S}}(\pi, A) P(A)$.

An important question we would like to answer is: "how can we characterize the capacity of the mobile ad hoc network under a scheduling policy $S$ (or $\hat{S}$ )?" To this end we need to spend few words on the routing strategy employed to transfer data through the network. The more general and abstract way to define a routing strategy is to specify quantities $f_{i j}^{s d} \in[0,1]$ denoting the average fraction of traffic from node $s$ to node $d$, which is routed through link $(i, j)$, i.e. $j$ follows $i$ as relay node [19]; $f_{i i}^{s d}=0$ by construction. The above quantities $f_{i j}^{s d}$ must satisfy the following well known flow conservation constraints:

$$
\sum_{i} f_{i j}^{s d}-\sum_{k} f_{j k}^{s d}=\left\{\begin{array}{cc}
1 & \text { for } j=d \\
0 & \text { for } j \neq d \text { and } j \neq s \\
-1 & \text { for } j=s
\end{array}\right.
$$

A routing strategy specified by a set of $f_{i j}^{s d}$ satisfying (1) can be easily implemented by the following simple hop-byhop routing algorithm $\mathcal{R}$ : every node $i$ in the network, upon reception of new data from source $s$, destined to $d$, routes them by selecting node $j$ as next hop with probability $f_{i j}^{s d} / \sum_{k} f_{i k}^{s d}$.

\section{Traffic sustainability and capacity region}

In this subsection we analyze the performance of a mobile ad hoc network comprising $n$ users, obtaining a precise characterization of its capacity region. We emphasize that our results are fairly general since only stationarity and ergodicity of traffic and mobility processes are required. We remark that, in our framework, the nodes movements are not constrained to be independent of each other.

Definition 1: We denote with $Z(t)$ the network backlog, that is, the amount of traffic (in bits) already generated by sources that has not yet been delivered to destinations at time $t$.

Definition 2: Traffic $\boldsymbol{\Lambda}$ is sustainable if there exists a scheduling policy $S$ and a routing strategy $\mathcal{R}$, such that: $\lim \sup _{t \rightarrow \infty} Z(t) / t=0$ w.p.1.

Definition 3: Traffic $\Lambda$ is strongly sustainable if there exists a simple scheduling policy $\hat{S}$ and a routing strategy $\mathcal{R}$, such that: $\lim \sup _{t \rightarrow \infty} Z(t) / t=0$ w.p. 1 .

We are now in a position to state our first result:

Theorem 1: A mobile ad hoc network sustains a traffic $\Lambda$, if a scheduling policy $S$ and a routing strategy $\mathcal{R}$ can be found such that:

$$
\sum_{s d} \lambda_{s d} f_{i j}^{s d} \leq \mu_{i j}^{S} \quad \forall i, j
$$

Moreover, if a simple scheduling policy $\hat{S}$ and a routing strategy $\mathcal{R}$ can be found such that:

$$
\sum_{s d} \lambda_{s d} f_{i j}^{s d} \leq \mu_{i j}^{\hat{S}} \quad \forall i, j
$$

the mobile ad hoc network strongly sustains traffic $\boldsymbol{\Lambda}$. Proof: The dynamics of the system can be described by a network of queues representing the evolution of the backlog at different nodes. We suppose that every node $i$ is equipped with $n-1$ separate transmission queues, each one storing data to be routed through a different node $j$. Upon reception, new data are immediately routed according to policy $\mathcal{R}$ and enqueued in the transmission queue associated to the next hop. Transmission queues are served at fixed rate $r$ according to a FCFS service policy, during the periods of activity of the corresponding link $(i, j)$. Note that, by construction, the average service rate in bit/s of the transmission queue of link $(i, j)$ is $\mu_{i j}^{S}$. The network of queues describing the system falls in the class of generalized Kelly networks, which are stable under the condition that no queues are overloaded [20]. Being, by construction, the load at the queue of link $(i, j)$ equal to $\sum_{s d} \lambda_{s d} f_{i j}^{s d} / \mu_{i j}^{S} \leq 1$, the assert follows immediately ${ }^{4}$.

As a corollary, we get a strict characterization of the traffic matrices which are strongly sustainable:

Proposition 1: A traffic matrix $\boldsymbol{\Lambda}=\left[\lambda_{s d}\right]$ is strongly sustainable iff a set of $f_{i j}^{s d} \in[0,1], \forall i, j, s, d$ and $p(\pi, A) \in[0,1]$ $\forall A, \pi \in A$ can be found satisfying the following equations:

$$
\begin{cases}f_{i j}^{s d} \text { satisfies (1) } & \forall i, j, s, d \\ \sum_{\pi \in A} p(\pi, A)=1 & \forall A \in \Pi \\ \sum_{s d} \lambda_{s d} f_{i j}^{s d} \leq r \sum_{A \in \Pi} \sum_{\pi \in A} \mathbb{I}_{(i, j) \in \pi} p(\pi, A) P(A) & \forall i, j\end{cases}
$$

Definition 4: The capacity region of the mobile ad hoc network is the set of all sustainable traffic matrices.

Definition 5: The restricted capacity region of the mobile ad hoc network is the set of all strongly sustainable traffic matrices.

Note that the restricted capacity region, by construction, depends on nodes mobility only via the joint stationary distribution of nodes. We can now state the following fundamental result:

Theorem 2: If traffic matrix $\Lambda$ is sustainable, then it is also strongly sustainable.

Proof: Let $S$ be the stationary and ergodic scheduling policy which sustains $\Lambda$. Define for every configuration $A$ and every $\pi \in A$ :

$$
\begin{aligned}
q_{S}(\pi, A)=\operatorname{Pr}\left\{\pi^{S}(t)=\pi \mid X(t) \in \mathcal{I}^{-1}(A)\right\}= \\
\lim _{t \rightarrow \infty} \frac{1}{t} \int_{0}^{t} \mathbb{I}_{\pi^{S}(\tau)=\pi \mid X(\tau) \in \mathcal{I}^{-1}(A)} d \tau \quad \text { w.p. } 1
\end{aligned}
$$

Due to the ergodicity of the mobility process and of the scheduling policy, the above quantities are well defined. It is immediate to verify that: $\sum_{\pi \in A} q_{S}(\pi, A)=1$. Thus considering a stationary, simple scheduling policy $\hat{S}$ such that $p_{\hat{S}}(\pi, A)=q_{S}(\pi, A)$, it follows, by construction: $\mu_{i j}^{S}=\mu_{i j}^{\hat{S}}$ $\forall i, j$.

The previous result has three significant implications: i) the class of simple policies achieves maximum throughput, i.e., no gain in terms of throughput can be obtained by adopting complex scheduling policies that select transmission configurations by considering dynamical variables such as instantaneous queues lengths, age of stored information at the nodes, etc; ii) a tight characterization of the capacity region is provided by Proposition 1 ; iii) the capacity region depends on the mobility process only through the joint-stationary distribution of nodes. This result extends and generalizes recent findings in [8]. At last,

${ }^{4}$ When $\sum_{s d} \lambda_{s d} f_{i j}^{s d} / \mu_{i j}^{S}=1$ the concept of stability is weak. 
Corollary 1: The capacity region of an ad hoc wireless network with mobile nodes is convex.

The proof is reported in [21].

\section{Contact graph: throughput and routing}

To understand the relationship between the scheduling policy and the routing strategy, we first need to characterize which traffic patterns are sustainable by employing an assigned scheduling policy $S$. Observe that the capacities ${ }^{5} \mu_{i j}$ associated to the communication links are univocally determined, once the scheduling policy $S$ has been selected. Thus a (capacitated) graph $G(\mathcal{V}, \mathcal{E})$ whose vertices correspond to network nodes and capacitated edges correspond to communication links, fully characterizes the mobile ad hoc network adopting $S$. In the following we refer to $G(\mathcal{V}, \mathcal{E})$ with the term contact graph. Therefore, the routing problem through the mobile ad hoc network adopting $S$ can be formalized in terms of a multicommodity flow problem on the contact graph.

Proposition 2: A traffic matrix $\Lambda=\left[\lambda_{s d}\right]$ can be sustained employing a policy $S$ iff the multi-commodity flow problem defined by (1) and (2), where communication link capacities are determined by $S$, admits a feasible solution. In such a case the set of variables $f_{i j}^{s d}$ univocally defines the routing strategy $\mathcal{R}$.

An alternative, partial characterization of the sustainable region achievable by scheduling policy $S$ (i.e. the set of $\boldsymbol{\Lambda}=$ $\left[\lambda_{s d}\right]$ that can be sustained employing $S$ ) can be provided in terms of the capacities associated to cuts of the contact graph.

Proposition 3: Traffic $\boldsymbol{\Lambda}=\left[\lambda_{s d}\right]$ is sustainable by policy $S$ only if, for any partition $(D, \bar{D})$ of the nodes, it results:

$$
\sum_{s \in \bar{D}} \sum_{d \in D} \lambda_{s d} \leq \sum_{s \in \bar{D}} \sum_{d \in D} \mu_{s d}
$$

For undirected Graphs $G(\mathcal{V}, \mathcal{E})$, it was proven in [22] that traffic $\Lambda$ is guaranteed to be sustainable if the ratio between the minimum value of the r.h.s. and the maximum value of the 1.h.s. is $\omega(\log m)$ being $m$ the number of flows. ${ }^{6}$

Consider a network adopting a scheduling policy $S$ and a routing strategy $\mathcal{R}$, under a sustainable traffic pattern $\Lambda$. Let $\nu$ be the network throughput, equal, by definition, to the offered network load $\nu=\sum_{s d} \lambda_{s d}$, and $|\pi(t)|$ be the size of $\pi(t)$ (i.e., the number of parallel transmissions enabled by $S$ at time $t$ ); it results:

$$
E[|\pi(t)|]=E\left[\sum_{i j} \mathbb{I}_{(i, j) \in \pi(t)}\right]=\sum_{i j} \frac{\mu_{i j}}{r}
$$

Let $C$ be the average aggregate transmission rate over all of the links; by construction:

$$
C=\sum_{s d} \lambda_{s d} \sum_{i j} f_{i j}^{s d} \leq r E[|\pi(t)|]
$$

The ratio $h_{\text {ave }}=C / \nu$ represents the average number of times that data are transmitted in the network; thus $h_{\text {ave }}$ is the

\footnotetext{
${ }^{5}$ To simplify the notation we omit, in this section, the explicit dependency from the scheduling policy $S$.

${ }^{6}$ Given two functions $f(n) \geq 0$ and $g(n) \geq 0: f(n)=$ $o(g(n))$ means $\lim _{n \rightarrow \infty} f(n) / g(n)=0 ; f(n)=O(g(n))$ mean $\limsup _{n \rightarrow \infty} f(n) / g(n)=c<\infty ; f(n)=\omega(g(n))$ is equivalent to $g(n)=o(f(n)) ; f(n)=\Omega(g(n))$ is equivalent to $g(n)=O(f(n))$ finally, $f(n)=\Theta(g(n))$ means $f(n)=O(g(n))$ and $g(n)=O(f(n))$.
}

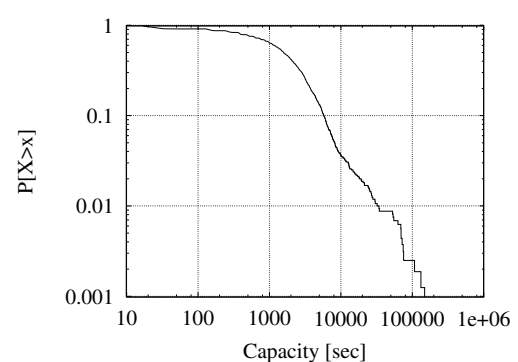

Fig. 1. Tail distribution function of the edge capacities

average length of the paths followed by information flows, expressed in number of hops. The following relationship is of immediate verification: $\left.\nu h_{\text {ave }} \leq E[\mid \pi(t)) \mid\right] r$.

In general, to efficiently exploit the network bandwidth, the routing strategy should minimize $h_{\text {ave }}$. This consideration justifies the fact that shortest path routing approaches have been widely used in several application contexts related to computer communications. In the context of mobile ad hoc networks, the 2-hop routing strategy proposed in [2] has gained a wide popularity; according to this strategy, data are delivered from source $s$ to destination $d$ either through the direct communication link, or through routes $s \rightarrow k \rightarrow d$, using every other node $k$ of the network as relay. Although the 2-hop routing strategy is appealing because of its simplicity, in general it does not allow to optimally exploit the network bandwidth, possibly causing a reduction of the sustainability region achievable by the scheduling policy, as we show in the following section.

\section{E. Capacity and routing in an experimental mobile network}

We have analyzed the traces collected during the iMotes experiment that took place during Infocom 2005 conference [3]. At that time, a set of 41 small intelligent network devices (implemented through iMotes) were carried in the pocket by some volunteers attending the conference; the iMotes had small radio range and, thanks to the mobility of the person, could contact other iMotes. The publicly available traces of the experiment [3] provide the radio contact durations (measured in seconds) between any two iMotes ${ }^{7}$; contact durations can be seen as an indirect measure of the capacity between nodes.

The resulting contact graph exhibits almost the maximum clustering (about $95 \%$ of all possible edges exists) and thus it looks like a fully connected graph. However it is important to observe that, as already pointed out in [3], the distribution of the edge capacities is characterized by a heavy tail, as shown in Fig. 1. Hence the contact graph contains significant asymmetries and inhomogeneous capacities. Fig. 2 shows visually the contact graph partitioned in three subgraphs: the left subgraph contains all the edges with small capacity (less than $500 \mathrm{sec}$ ), the central subgraph (almost fully connected) contains medium capacity edges (between 500 and 5,000 sec) and the right subgraph shows high capacity edges (more than $5,000 \mathrm{sec}$ ) in which we also highlighted in bold all the edges with capacity larger than 50,000 sec. Observe that, even if

\footnotetext{
${ }^{7}$ The traces refer also to some external devices, but since the information about the contacts among such external devices is not available, we restricted our analysis to iMotes only.
} 

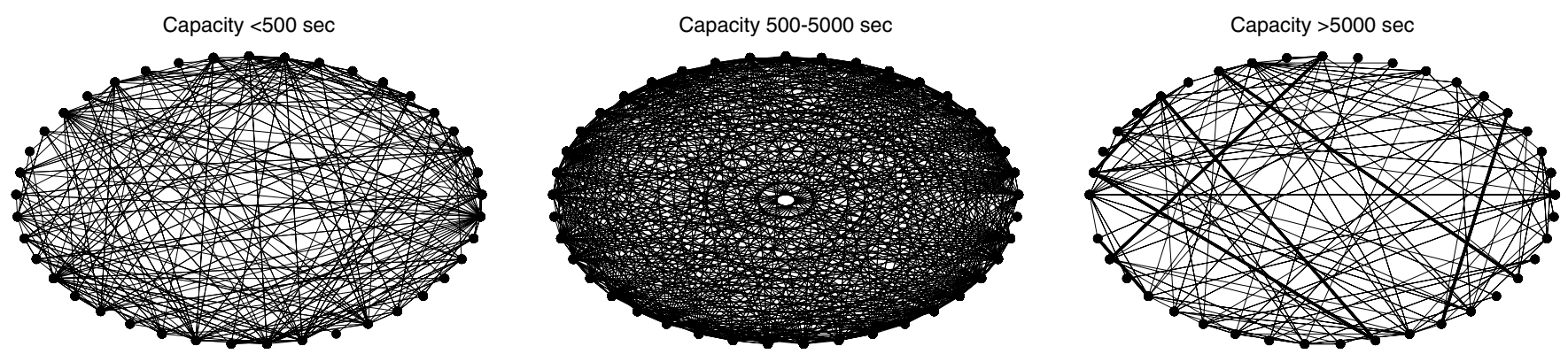

Fig. 2. Contact graph of Infocom-iMotes experiment, subdivided in three subgraphs (bold lines on the right represent edges with capacity larger than 50,000 $\mathrm{sec}$ ). Edges of the contact graph are distributed among the three subgraphs according to the following proportion: $22.3 \%, 64.5 \%, 13.2 \%$. In terms of aggregate transport capability, the contribution of the three subgraphs is: $1.3 \%, 40.3 \%, 58.4 \%$ respectively.

\begin{tabular}{|c|c|c|}
\hline Traffic scenario & Maximum aggregate capacity & Ave. number of hops \\
\hline$m D C$ & $1.75610^{6} \mathrm{sec}$ & 2.605 \\
$M D C$ & $3.00810^{6} \mathrm{sec}$ & 1.692 \\
\hline
\end{tabular}

TABLE I

OPTIMAL CAPACITY AND ROUTING FOR INFOCOM-IMOTES EXPERIMENT

only $13.2 \%$ of the edges have high capacity, they contribute $58.4 \%$ of the overall transport capability.

Through the solution of the multi-commodity flow problem previously described, maximizing $\sum_{s d} \lambda_{s d}$, we have also evaluated the maximum achievable throughput for two specific traffic scenarios, in which each node of the network is origin and destination of a single traffic flow. The 41 node pairs that exchange traffic in the two scenarios (named minimum Direct Capacity - $m D C$ and Maximum Direct Capacity - $M D C$ respectively), has been selected so to minimize/maximize the sum of capacities associated to the direct communication links between sources and destinations.

Table I shows the aggregate maximum throughput and the average number of hops for the corresponding optimal routing strategy. In both cases we observed routes longer than 2 hops; in particular, for the $m D C$ scenario, even the average number of hops is significantly larger than 2 .

\section{ASYMPTOTIC ANALYSIS FOR $n \rightarrow \infty$}

To analyze asymptotic properties of the network when the number of nodes becomes large, we progressively increase the number of nodes $n$ in the network, generating a sequence of systems indexed by $n$. In each system, nodes are moving in a closed connected and finite region $\mathcal{A}$ (for simplicity we assume $|\mathcal{A}|=1$ ), according to stationary and ergodic mobility processes ${ }^{8}$. We denote with $\mathbf{X}^{(n)}(t) \in \mathbb{R}^{2 n}$ the vector representing the nodes positions at time $t$ in system $n$. We suppose that the following "continuity" property holds over the sequence of systems.

Property 1: Given any finite $M$-uple of mobiles $\left(i_{1}, \ldots i_{M}\right)$ and defined for any $i_{m}$, (with $\left.1 \leq m \leq M\right)$ a sequence of measurable sets $B_{m}^{(n)}$ with $B_{m}^{(\bar{n})} \downarrow B_{m}$ (or $\left.B_{m}^{(n)} \uparrow B_{m}\right)$, there must exist:

$$
\lim _{n \rightarrow \infty} E\left[\mathbb{I}_{\left(\cap_{m} X_{i_{m}}^{(n)}(t) \in B_{m}^{(n)}\right)}\right]
$$

\footnotetext{
${ }^{8}$ In the following, to simplify the notation, whenever strictly unnecessary we omit the dependency on $n$.
}

that represents the asymptotic probability that mobiles $\left(i_{1}, \ldots, i_{M}\right)$ fall in $B=\left(B_{1}, \ldots, B_{M}\right)$.

Essentially we are interested in establishing how the network capacity scales with $n$, under non-uniform mobility models. Without lack of generality we normalize the transmission rate $r$ to 1 and on the analogy of previous work we restrict our investigation to uniform permutation traffic patterns, i.e., traffic patterns in which every node is origin and destination of a single traffic flow with average rate $\lambda$; however, as immediate consequence of the capacity region convexity (Corollary 1), all of our asymptotic results can be extended to more general traffic patterns.

More specifically, we say that the asymptotic per-node capacity (or per-node maximum achievable throughput) of the system is $\Theta(f(n))$ if, given a sequence of uniform permutation traffic patterns with average rate $\lambda^{(n)}=f(n)$, there exist two constants $c<c^{\prime}$ such that both the following properties hold:

$$
\lim _{n \rightarrow \infty} \operatorname{Pr}\left\{c \lambda^{(n)} \text { is sustainable }\right\}=1
$$

and

$$
\lim _{n \rightarrow \infty} \operatorname{Pr}\left\{c^{\prime} \lambda^{(n)} \text { is sustainable }\right\}<1
$$

Equivalently, we say in this case that the network capacity (or maximum network throughput) is $\Theta(n f(n))$.

Given a point $X_{0} \in A$, we define the local asymptotic density of nodes in $X_{0}$ as:

$$
\rho\left(X_{0}\right)=\lim _{n \rightarrow \infty} \sum_{i=1}^{n} E\left[\mathbb{I}_{X_{i}^{(n)}(t) \in B\left(X_{0}, 1 / \sqrt{n}\right)}\right]
$$

where $B\left(X_{0}, 1 / \sqrt{n}\right)$ is the disk centered in $X_{0}$, of radius $1 / \sqrt{n}$.

\section{A. The impact of transmission range}

First we discuss the impact of the transmission range associated to node-to-node communications selected by the scheduling policies on the achievable asymptotic throughput as $n \rightarrow \infty$.

Theorem 3: Given a network comprising $n$ nodes. Let $S^{(n)}$ be the associated scheduling policy. If $S^{(n)}$ achieves asymptotically a network throughput $\nu(n)=\Theta(f(n))$, then only a negligible amount of traffic $o(f(n))$ is transferred 
exploiting node-to-node communications whose transmission range is $\omega\left(\frac{1}{\sqrt{f(n)}}\right)$.

The proof is reported in [21].

The previous theorem essentially states that throughput $\nu(n)$ is achievable only by those scheduling policies selecting with high probability (w.h.p.) (i.e. with a probability converging to 1 as $n \rightarrow \infty)$ transmitters and receivers pairs whose distance is $O\left(\frac{1}{\sqrt{\nu(n)}}\right)$. Hence, the scheduling policies achieving a network throughput $\nu(n)=\Theta(n)$ must exploit transmission ranges within $O\left(\frac{1}{\sqrt{n}}\right)$. Furthermore,

Theorem 4: Given a network comprising $n$ nodes. If (i) node movements are independent and (ii) the asymptotic node density is finite and different from zero at every point $X_{0} \in A$, i.e. there exist $\rho_{1}, \rho_{2}$ such that:

$$
0<\rho_{1} \leq \rho\left(X_{0}\right) \leq \rho_{2}<\infty \quad \text { for every } X_{0} \in A
$$

the asymptotic capacity is achievable by the class of scheduling policies which forces the transmission range to be w.h.p. $O\left(\frac{\log (n)}{\sqrt{n}}\right)$.

The proof is reported in [21].

The above results concerning the transmissions ranges essentially provide the guidelines to the design of throughput efficient scheduling policies, indicating that transmission ranges should be reduced as much as possible. This finding is in line with observations in [1], [2] for particular cases. A generic communication $(i, j)$ occurring at time $t$ interferes with possible communications involving nodes within the transmission range used; thus, by reducing the transmission range of nodes, the transmission parallelism $|\pi(t)|$ is increased on average, resulting in a maximization of the global transport capability (5). Instead, wherever the local asymptotic density of nodes is finite and non null, the selection of a node-to-node communication with range $\omega\left(\frac{1}{\sqrt{n}}\right)$ blocks on average an (asymptotically) infinite number of other potential communications. So the choice of allowing communications with transmission ranges $\omega\left(\frac{1}{\sqrt{n}}\right)$, unless strictly necessary (as for the special case of static nodes), leads to a suboptimal exploitation of the system bandwidth, resulting in a global throughput reduction.

For the above reasons, in the following we mainly focus our investigation on the class of scheduling policies forcing transmission ranges to be $O\left(\frac{1}{\sqrt{n}}\right)$.

\section{B. The $S^{*}$ scheduling policy}

Following the above described guidelines, we propose the following stateless and memoryless scheduling policy:

Definition 6: Given a network comprising $n$ nodes, policy $S^{*}$ schedules transmission between $i$ and node $j$ under the following condition:

$$
\min \left(d_{k j}(t), d_{k i}(t)\right)>(1+\Delta) d_{i j}(t) \quad \text { for } \Delta>0
$$

for every other node $k$ in the network (regardless of node $k$ activity). Notice that, under scheduling policy $S^{*}$, the transmission bandwidth between $i$ and $j$ is equally shared in the two directions.
Long term capacities $\mu_{i j}^{*}$ achieved by $S^{*}$ can be expressed as function of the joint stationary spatial distribution of mobiles according to:

$$
\begin{aligned}
\mu_{i j}^{*} & =\lim _{t \rightarrow \infty} \frac{1}{2 t} \int_{0}^{t} \mathbb{I}_{\left(\cap_{k \neq i, j} \min \left(d_{k j}(\tau), d_{k i}(\tau)\right)>(1+\Delta) d_{i j}(\tau)\right)} d \tau \\
& =\frac{1}{2} E\left[\mathbb{I}_{\left(\cap_{k \neq i, j} \min \left(d_{k j}(t), d_{k i}(t)\right)>(1+\Delta) d_{i j}(t)\right)}\right] \quad \text { w.p. } 1
\end{aligned}
$$

When movements of mobiles are independent, $\mu_{i j}^{*}$ can be obtained as function of marginal spatial distribution of individual nodes:

$$
\begin{array}{r}
\mu_{i j}^{*}=\frac{1}{2} \int_{X_{i} \in A} \int_{X_{j} \in A}\left[\prod_{k \neq i, j} \int_{X \in A_{\Delta}\left(X_{i}, X_{j}\right)} d F_{k}(X)\right] \\
d F_{j}\left(X_{j}\right) d F_{i}\left(X_{i}\right)
\end{array}
$$

being $F_{i}(X)$ the spatial Distribution of node $i$ and $A_{\Delta}\left(X_{i}, X_{j}\right)$ the area outside the transmission range used by $i$ to communicate with $j: A_{\Delta}\left(X_{i}, X_{j}\right)=\{X: \min (\| X-$ $\left.\left.X_{j}\left\|_{2},\right\| X-X_{i} \|_{2}\right)>(1+\Delta)\left\|X_{i}-X_{j}\right\|_{2}\right\}$.

Theorem 5: Under the assumption that mobiles movements are independent and that (6) holds, w.h.p. the transmission ranges selected by $S^{*}$ are $O(1 / \sqrt{n})$. In addition, for any pair of nodes $(i, j)$ and any finite $c>0$, it results:

$$
\mu_{i j}^{*}=\Theta\left(\operatorname{Pr}\left\{d_{i j} \leq \frac{c}{\sqrt{n}}\right\}\right)
$$

The proof is reported in appendix I.

As a consequence, no sequence of scheduling policies $S(n)$ forcing the transmission ranges to be $O\left(\frac{1}{\sqrt{n}}\right)$ can achieve an asymptotically higher throughput than sequence of policies $S^{*}(n)$. In addition, by applying Theorem 3 and Theorem 5, we can conclude that:

Corollary 2: If $S^{*}(n)$ achieves a network throughput $o(n)$, then no other sequence of policies exists that achieves an asymptotic network throughput $\Theta(n)$.

\section{AppliCATIONS OF THE ASYMPTOTIC ANALYSiS}

\section{A. Independent mobile nodes with uniform distribution}

We revisit the case in which nodes move independently and uniformly over a closed domain $\mathcal{A}$ as in [2], showing that $S^{*}$ achieves $\Theta(n)$ network throughput. First, observe that by symmetry all the node-to-node capacities $\mu_{i j}^{*}$ must be equal. Moreover, since by construction $\sum_{j} \mu_{i j}^{*} \leq 1$, it necessarily follows $\mu_{i j}^{*} \leq \frac{1}{n-1}$. To avoid edge effects (which however can be proved to be irrelevant along the lines of [1]) we suppose $\mathcal{A}$ to be the spheric surface of unit area. We apply (7) to evaluate the capacity between any two mobiles $i$ and $j$. We denote with $\phi$ the angle described by the positions $X_{i}$ and $X_{j}$ of nodes $i$ and $j$, whose distance results to be $\phi /(2 \sqrt{\pi})$. Using simple geometrical arguments we have:

$$
\frac{1}{4} \int_{0}^{\frac{\pi}{2(1+\Delta)}} \sin (\phi)[\cos ((1+\Delta) \phi)]^{n-2} d \phi \leq \mu_{i j}^{*} \leq \frac{1}{n-1}
$$

Note that, by restricting the integration domain to interval $\left[0, \frac{1}{\sqrt{n}(1+\Delta)}\right]$, and observing that for small values of $\phi \geq 0$, $\sin (\phi) \geq \phi+\phi^{3} / 3$ and $\cos (\phi) \geq 1-\phi^{2} / 2$, it results:

$$
\begin{array}{r}
\mu_{i j}^{*} \geq \frac{1}{4} \int_{0}^{\frac{1}{\sqrt{n}(1+\Delta)}}\left[\phi-\frac{\phi^{3}}{3}\right]\left[1-\frac{[(1+\Delta) \phi]^{2}}{2}\right]^{n-2} d \phi= \\
\frac{1}{8 n(1+\Delta)^{2}}+o\left(\frac{1}{n}\right)
\end{array}
$$


which proves that $\mu_{i j}^{*}=\Theta(1 / n)$. At last, to show that the network maximum throughput is $\Theta(n)(\Theta(1)$ per node), as found in [2], we select a 2-hop routing strategy. In this case, every communication link $(i, j)$ is traversed by at most two traffic flows (the flow originated at $i$ and the traffic flow destined to $j$ ). Thus, by dividing equally the link capacity among the two traffic flows, the total capacity on paths from source $s$ to destination $d$, exclusively devoted to the transport of traffic flow $(s, d)$, is: $\mu_{s d}^{*}+\frac{1}{2} \sum_{k \neq s, d} \min \left(\mu_{s k}^{*}, \mu_{k d}^{*}\right)=$ $\Theta\left(\frac{1}{n}\right)+(n-2) \Theta\left(\frac{1}{n}\right)=\Theta(1)$.

\section{B. Examples of non-uniform spatial distributions}

Now, we explicitly compute the asymptotic network capacity (as $n \rightarrow \infty$ ), achievable by scheduling policies forcing the transmission range of communications to be $O\left(\frac{1}{\sqrt{n}}\right)$, in a few interesting cases in which the spatial distribution of each mobile node is non-uniform over the network area. For simplicity, we assume that nodes move independently of each other.

Thanks to Theorem 5, within the above mentioned class of scheduling policies, maximum throughput is achieved by policy $S^{*}(n)$, according to which the communication link capacity $\mu_{i j}^{*(n)}$ between any pair of nodes is:

$$
\mu_{i j}^{*(n)}=\Theta\left(\int_{X_{i} \in \mathcal{A}} \int_{X_{j} \in \mathcal{A}} \mathbb{I}_{d_{i j}<R_{n}} d F_{j}^{(n)}\left(X_{j}\right) d F_{i}^{(n)}\left(X_{i}\right)\right)
$$

being $R_{n}=\frac{1}{\sqrt{n}}$. We consider a class of node spatial distributions characterized as follows: each node selects a point as its 'home point', chosen uniformly at random within the area. The probability that it visits another point at distance $d$ from its home point is then given by an assigned distribution $g(d)$. Notice that the networks resulting from our model are random in nature. Therefore, to precisely characterize the asymptotic network capacity as $n \rightarrow \infty$, we rely on the notion of typical configurations introduced in [4]. Basically, our results are meant to hold with high probability.

To simplify the analysis, we take $\mathcal{A}$ to be a square torus of unit area ${ }^{9}$. We consider a coordinate system centered at the home-point of a node, and express the probability that the node visits point $(x, y)$ in the form $g(|x|) g(|y|)$, with $-1 / 2 \leq x, y \leq 1 / 2$. One can show that the capacity obtained under this model is the same (in order sense) as that achieved in case the spatial distribution has a circular symmetry around the home point. Furthermore, we approximate the probability that nodes $i$ and $j$ are within euclidean distance $R_{n}$, with the probability (equivalent in order sense) that the absolute difference of their coordinates along the $x$ and $y$ axes are jointly smaller than $R_{n}$. This allows us to further simplify the evaluation of $\mu_{i j}^{*}$, since we have in this case $\mu_{i j}^{*}=\Theta\left(G\left(d_{x}\right) G\left(d_{y}\right)\right)$, where $d_{x}$ and $d_{y}$ are the distance components between the home points of $i$ and $j$, along the $x$ and $y$ axes, respectively. Function $G(d)$ is the probability that two nodes, moving along a circle of length 1 with distribution $g(d)$ around their home points $(0 \leq d \leq 1 / 2)$, happen to be

\footnotetext{
${ }^{9}$ The same asymptotic results hold, in order sense, on the surface of a sphere, or on any compact set in $\mathbb{R}^{2}$.
}
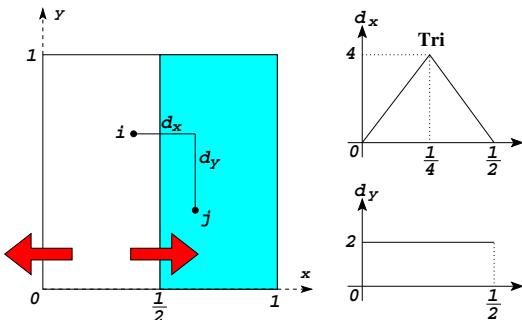

Fig. 3. Partition of the network area and distributions of the distances $d_{x}$ and $d_{y}$

within distance $R_{n}$ of each other, being $d$ the distance between their home points along the same circle.

Once we have computed $G(d)$, the network capacity can be evaluated resorting to Proposition 3 applied to the contact graph of a typical network configuration.

Under the assumption that $G(d)$ is non-increasing with $d$, the asymptotic critical cut (i.e., the cut which determines the network capacity for $n \rightarrow \infty$ ) is obtained, in typical network configurations, partitioning the $n$ nodes into two sets of cardinality $n / 2+o(n)$, such that the the sum of the distances between the home points of any pair of nodes belonging to different sets is maximized. In the case of a torus, such critical cuts are obtained dividing the area into two halves of size $\frac{1}{2} \times 1$ (through a physical linear cut), and grouping in one set all nodes having their home point in the same half of the area ${ }^{10}$ (see Figure 3).

The distance distribution between two generic home points located on different halves of the torus can be characterized as follows: the component $d_{y}$ along the $y$ axes is uniformly distributed over the interval $[0,1 / 2]$. The component $d_{x}$ along the $x$ axes has, instead, the triangular distribution $\operatorname{Tri}(x)$ depicted in Figure 3. In typical network configurations, the traffic flows $(s, d)$ traversing the physical cut from left to right (i.e. traffic flows $(s, d)$ such that the home point of $s$ is in the left half and the one of $d$ in the right half) are $n / 4+o(n)$, while the edges across the same cut are $n^{2} / 4+o\left(n^{2}\right)$ (from left to right). Moreover, the expected fraction of edges connecting pairs of nodes with given values of $d_{x}$ and $d_{y}$ is asymptotically provided by the above distributions of $d_{x}$ and $d_{y}$. It turns out that the asymptotic network throughput $\nu(n)$, as $n \rightarrow \infty$, satisfies:

$$
\nu(n)=\omega\left(\frac{\bar{\nu}(n)}{\log (n)}\right) \quad \nu(n)=O(\bar{\nu}(n))
$$

being:

$$
\bar{\nu}(n)=n \Theta\left(n \int_{0}^{1 / 2} 2 G(y) \mathrm{d} y \int_{0}^{1 / 2} \operatorname{Tri}(x) G(x) \mathrm{d} x\right)
$$

We now compute the function $G(d)$ and the resulting network throughput in a few interesting cases.

1) Uniform distribution over a restricted domain: First, we assume $g(d)$ to be constant over the interval $[0, q]$ and zero for $d>q$, where $q \leq 1 / 2$. This corresponds, on the surface of the torus, to a mobility model according to which a node uniformly visits a square of edge $2 q$ centered at his home point, and never goes outside of it. When $q=R_{n}$ nodes

\footnotetext{
${ }^{10}$ The considered critical cut is just one of many equivalent cuts.
} 
can be considered to be fixed (Gupta-Kumar case), whereas for $q=1 / 2$ we obtain the uniform distribution over the whole area (Grossglauser-Tse case). Therefore it is interesting to explore how the network capacity varies as we increase $q$ from $R_{n}$ to $1 / 2$. In particular, we let $q=\Theta\left(n^{\delta}\right)$, and vary $\delta$ from $-1 / 2$ (Gupta-Kumar case) to 0 (Grossglauser-Tse case). Notice that, as long as $\delta$ is strictly larger than $-1 / 2$, the network is connected almost surely even if transmissions are constrained to be $\Theta(1 / \sqrt{n})$ : indeed, by allowing nodes to reach a minimum distance from their home points, of order $\Omega(\sqrt{\log n / n})$, connectivity is no longer an issue as in the case of random networks with static nodes.

The computation of $G(d)$ is rather immediate in this case, It results: $G(d)=(2 q-d) \frac{R_{n}}{q^{2}}$, for $0<d<2 q$ (we assume that $q<1 / 4$ ), whereas $G(d)$ is identically zero for $2 q<d<1 / 2$. Plugging the above expression of $G(d)$ into (8) we obtain

$$
\bar{\nu}(n)=\Theta\left(n^{2} R_{n}^{2} q\right)=\Theta\left(n^{1+\delta}\right)
$$

Thus, as we vary $\delta$ from $-1 / 2$ to 0 , the network capacity varies from $\Theta(\sqrt{n})$ (Gupta-Kumar case) to $\Theta(n)$ (Grossglauser-Tse case).

2) Exponential distribution: The case of restricted mobility considered in the previous example is a trivial one, because the average number of hops required to reach a destination located at distance $\Theta(1)$ from the source (almost all connections) is inevitably $\Theta(1 / q)=\Theta\left(n^{-\delta}\right)$, hence it grows to infinity ( $\delta$ is negative), and the resulting network capacity has to scale down accordingly. A more interesting case is when $g(d)$ is non-zero over the entire space. This means that every node soon or later meets every other node (with probability one), thus one can apply, in principle, the two-hop relay scheme of Grossglauser-Tse, minimizing the number of hops while allowing for communication diversity. Unfortunately, the achievable network capacity is not always $\Theta(n)$ even if nodes "fill the space over time". As an example, we consider the case in which $g(d)$ decays exponentially with $d$, with parameter $\gamma$. More specifically, since the area is finite, we consider the truncated exponential $g(d)=\gamma e^{-\gamma d} /\left(2\left(1-e^{-\gamma / 2}\right)\right)$. The complete expression of $G(d)$ is

$$
\begin{gathered}
G(d)=\frac{e^{-\left(d+R_{n}\right) \gamma}\left[e^{\gamma}\left(e^{2 R_{n} \gamma}-1\right)(1+d \gamma)+\right.}{4\left(e^{\gamma / 2}-1\right)^{2}} \\
\left.+e^{\left(d-R_{n}\right) \gamma}\left(2 R_{n} \gamma-d \gamma+3\right)+e^{\left(d+R_{n}\right) \gamma}\left(2 R_{n} \gamma+d \gamma-3\right)\right]
\end{gathered}
$$

Substituting the above expression into (8) we obtain

$$
\bar{\nu}(n)=\Theta\left(\frac{n^{2} R_{n}^{2}}{\gamma}\right)
$$

which is formally identical to (9) provided that $\gamma=1 / q$. Thus, by letting $1 / \gamma=n^{\delta}$, with $-1 / 2<\delta<0$, we obtain a network capacity $\Theta\left(n^{1+\delta}\right)$.

We have repeated the computation in case of a truncated Pareto distribution defined over $\left[R_{n}, 1 / 2\right]$, obtaining a similar continuous variation of network capacity in between the extreme cases of Gupta-Kumar and Grossglauser-Tse as we vary the mean value of $g(d)$, this time through the power-law exponent.
3) Uni-dimensional mobility: Quite surprisingly, a network capacity of $\Theta(1)$ can be sustained even when nodes visit a small fraction of the entire network area. This has been already pointed out in [4], where the authors assume that nodes are constrained to move just along uni-dimensional paths. Our framework allows to recover and extend this result in a simple way. For simplicity, we assume that nodes are constrained to move either on a vertical or horizontal path (each with probability 1/2) passing through their home-point. As opposed to the uniform assumption adopted in [4], we allow the nodes to visit the points along their path according to a given distribution $g(d)$ of the distance from the home point, which is assumed to be non-increasing with $d$. The dominant contribution to network capacity is provided by communication links between nodes moving along orthogonal directions. Actually, contacts between a horizontal (vertical) node $i$ and a vertical (horizontal) node $j$, occur only when both nodes simultaneously fall within distance $R_{n}$ from the unique intersection point between their paths (see Fig. 3). Hence the capacity $\mu_{i j}^{*}$ of communication link $(i, j)$, is $\mu_{i j}^{*}=$ $\Theta\left(g\left(d_{x}\right) g\left(d_{y}\right) R_{n}^{2}\right)$, where the distributions of $d_{x}$ and $d_{y}$ are those reported in the right of Figure 3. Considering that $G(d)$ is $\Theta\left(g(d) R_{n}\right)$, and that the number of edges across the critical cut is $n^{2} / 8+o\left(n^{2}\right)$, by comparing with (8) we recognize that $\bar{\nu}(n)$ is the same as that achieved under a two-dimensional mobility pattern having the same distance distribution $g(d)$ along the two dimensions.

This fact allows us to make an interesting observation. Suppose that nodes are constrained to visit uniformly a fraction $n^{-\zeta}$ of the space, with $1 / 2<\zeta<1$, over a rectangular domain of minimum edge equal to $n^{-\frac{1}{2}}$, and random orientation. It turns out the the best solution in terms of network capacity is to stretch out the domain as much as possible, forming a rectangle $n^{-\frac{1}{2}} \times n^{\frac{1}{2}-\zeta}$, which achieves a capacity $n^{\frac{3}{2}-\zeta}$. The worst case instead is the square of edge $n^{-\frac{\zeta}{2}}$, which achieves a capacity $n^{1-\frac{\zeta}{2}}$. We conclude that the network capacity is significantly affected by the shape of the domain visited by the nodes. In this sense, uni-dimensional mobility over maximum length paths (as in [4]) can be considered to be a best-case scenario.

4) Multiple classes of nodes: Our technique based on the application of Proposition 3 over the typical contact graph provides quite a powerful and flexible tool to evaluate the network capacity in very general conditions. In particular, we can also mix different classes of users with different mobility patterns. As an example, we consider the case of $n^{\zeta}$ nodes visiting uniformly the entire area (class A nodes), with $0<\zeta<1$; the remaining $n-n^{\zeta}$ nodes (class $\mathrm{B}$ ) are assumed to move around a randomly located home-point with distribution $g(d)$. The average of $g(d)$ is $\Theta\left(n^{\delta}\right),(\delta<0)$, as in the previously considered examples. To evaluate the network capacity, we consider that the critical cut is traversed by: $n^{2 \zeta} / 4+o\left(n^{2 \zeta}\right)$ edges of capacity $\Theta\left(\frac{1}{n}\right)$ (among nodes of class A), $\left(n-n^{\zeta}\right)^{2} / 4+o\left(\left(n-n^{\zeta}\right)^{2}\right)$ edges of capacity $\Theta\left(n^{\delta-1}\right)$ (among nodes of class B), and $n^{\zeta}\left(n-n^{\zeta}\right) / 2+o\left(n^{\zeta}\left(n-n^{\zeta}\right)\right)$ edges of capacity $\Theta\left(\frac{1}{n}\right)$ (cross capacity A-B). We obtain a network capacity $\nu(n)=\Theta\left(n^{\zeta}+n^{1+\delta}\right)$. Thus, to have any impact on network capacity (in order sense), the fraction $n^{\zeta}$ 
of fully mobile nodes of class $A$ must satisfy $\zeta>\delta+1$.

\section{Conclusions}

In this paper we have considered an ad hoc wireless network composed of $n$ heterogeneous mobile nodes and proposed a general methodology that allows to precisely characterize its capacity region by considering the associated contact graph, highlighting several important structural properties of the system. We have, then, extended our study to the asymptotic regime (for $n \rightarrow \infty$ ) and obtained fundamental results of general validity. Finally we have computed the asymptotic capacity of an ad-hoc network under significant examples of non-uniform mobility models of the nodes. Our results show that under anisotropic mobility patterns network capacity may vary with continuity from $\Theta(\sqrt{n})$ (Gupta-Kumar case) to $\Theta(n)$ (Grossglauser-Tse case).

\section{REFERENCES}

[1] P. Gupta, P.R. Kumar, "The capacity of wireless networks", IEEE Trans. on Information Theory, vol. 46, n.2, pp. 388-404, Mar. 2000

[2] M. Grossglauser, D.N.C. Tse, "Mobility increases the capacity of ad hoc wireless networks", IEEE Trans. on Networking, vol. 10, n.2, pp. 477-486, Aug. 2002

[3] P. Hui, A. Chaintreau, J. Scott, R. Gass, J. Crowcroft, C. Diot, "Pocket Switched Networks and the Consequence of Human Mobility in Conference Environments", ACM WDTN, Philadelphia, PA, Aug. 2005

[4] S.N. Diggavi, M. Grossglauser, D.N.C. Tse, "Even one-dimensional mobility increases ad hoc wireless capacity", IEEE Trans. on Information Theory, vol. 51, n. 11, pp. 3947-3954, Nov. 2005

[5] S. Toumpis and A. Goldsmith, "Large wireless networks under fading, mobility, and delay constraints", IEEE INFOCOM, Hong Kong, China, Mar. 2004

[6] A.El Gamal, J. Mammen, B. Prabhakar, and D. Shah, "ThroughputDelay Trade-off in Wireless Networks", IEEE INFOCOM, Hong Kong, China, Mar. 2004

[7] G. Sharma, R. R. Mazumdar and N.B.Shroff, "Delay and Capacity Trade-offs in Mobile Ad Hoc Networks: A Global Perspective" IEEE INFOCOM, Barcelona, Spain, Apr. 2006

[8] A. Al Hanbali, A. A. Kherani, R. Groenevelt, P. Nain, E. Altman, "Impact of Mobility on the Performance of Relaying in Ad Hoc Networks," IEEE INFOCOM, Barcelona, Spain, Apr. 2006

[9] J. Mammen, D. Shah, "Throughput and Delay in Random Wireless Networks: 1-D Mobility is Just As Good As 2-D", IEEE ISIT, Chicago, IL, July 2004

[10] R. M. Moraes, H. R. Sadjadpour and J. J. Garcia-Luna Aceves, "MobilityCapacity-Delay Trade-off in Wireless Ad Hoc Networks," Elsevier Journal on ad hoc networks, July 2005

[11] Delay Tolerant Network Research Group (DTNRG): www . dtnrg.org

[12] S. R. Kulkarni, P. Viswanath, "A Deterministic Approach to Throughput Scaling in Wireless Networks," IEEE Trans. on Infornation Theory, vol. 50, no. 6 , pp. 1041-1049, June 2004

[13] S. Toumpis, "Capacity bounds for three classes of wireless networks: asymmetric, cluster, and hybrid", ACM MobiHoc, pp. 133-144, Tokyo, Japan, May 2004

[14] R. Madan, D. Shah, "Capacity-Delay Scaling in Arbitrary Wireless Networks", Allerton Conference, Monticello, IL, 2005

[15] U.C. Kozat and L. Tassiulas, "Throughput capacity of random ad hoc networks with infrastructure support" ACM MobiCom, pp. 55-65, San Diego, CA, Sep. 2003

[16] A. Agarwal and P. R. Kumar, "Capacity bounds for ad-hoc and hybrid wireless networks", ACM Computer Communications Review, vol. 34, no. 3, pp. 71-81, July 2004

[17] B.Liu, Z. Liu, and D. Towsley, "On the capacity of hybrid wireless networks", IEEE INFOCOM, vol. 2, pp. 1543-1552, San Francisco, CA, Apr. 2003

[18] S. R. Kulkarni, A. Reznik and S. Verdú, "A "Small World" Approach to Heterogeneous Networks," Commun. Inf. Syst., vol. 3, no. 4, pp. 325 348, Sep. 2004
[19] L. Fratta, M. Gerla, L. Kleinrock, "The Flow Deviation Method - An Approach to the Store-and-Forward Communication Network Design," Networks, vol. 3, pp. 97-133, 1973

[20] M. Bramson, "Convergence to Equilibrium for Fluid Models of FIFO Queueing Networks", Queueing Systems, vol. 22, pp. 5-45, 1996

[21] M. Garetto, P. Giaccone, E. Leonardi, "On the Capacity of Ad Hoc Wireless Networks Under General Node Mobility", Technical report, available at www.tlc-networks.polito.it/database

[22] Y. Aumann and Y. Rabani, "An $O(\log k)$ Approximate Min-Cut MaxFlow Theorem and Approximation Algorithm", SIAM Journal on Computing, vol. 27 , n. 1,1998

\section{APPENDIX I}

\section{PROOF OF THEOREM 5}

First we prove that for each $i$ and $j, \mu_{i j}^{*(n)}=\Theta\left(\operatorname{Pr}\left\{d_{i j}^{(n)} \leq\right.\right.$ $\left.\left.\frac{c}{\sqrt{n}}\right\}\right)$. Indeed, by construction:

$\operatorname{Pr}\left\{d_{i j}^{(n)} \leq \frac{c}{\sqrt{n}}\right\}=\int_{X_{i} \in \mathcal{A}} \int_{X_{j} \in B\left(X_{i}, c / \sqrt{n}\right)} d F_{j}^{(n)}\left(X_{j}\right) d F_{i}^{(n)}\left(X_{i}\right)$

while, by restricting the integration domain in (7):

$$
\begin{aligned}
& \left.\mu_{i j}^{*(n)} \geq \frac{1}{2} \int_{X_{i} \in A} \int_{X_{j} \in B\left(X_{i}, c / \sqrt{n}\right)} d F_{k}^{(n)}(X)\right] d F_{j}^{(n)}\left(X_{j}\right) d F_{i}^{(n)}\left(X_{i}\right) \\
& {\left[\prod_{k \neq i, j} \int_{X \in A_{\Delta}\left(X_{i}, X_{j}\right)}\right.}
\end{aligned}
$$

Thus to prove the assert, it is sufficient to show that $\lim _{n \rightarrow \infty} \prod_{k \neq i, j} E\left[\mathbb{I}_{X_{k}^{(n)}(t) \in A_{\Delta}\left(X_{i}, X_{j}\right)}\right]>\epsilon$ a.e. for some $\epsilon>0$. Note that $\lim _{n \rightarrow \infty} E\left[\mathbb{I}_{X_{i}^{(n)}(t) \in B\left(X_{0}, c / \sqrt{n}\right)}\right]$ represents the limiting probability of finding mobile $i$ at location $X_{0}$, i.e., by ergodicity, the limiting fraction of time that mobile $i$ spends still at location $X_{0}$. By construction, excluding at most a denumerable set of points $\left\{X_{m}\right\}, \forall X_{0} \notin\left\{X_{m}\right\}$, it results: $\lim _{n \rightarrow \infty} E\left[\mathbb{I}_{X_{i}^{(n)}(t) \in B\left(X_{0}, c / \sqrt{n}\right)}\right]=0$. Thus fixing $X_{i}$ with $X_{i} \neq X_{m}$, considering $X_{j} \in B\left(X_{i}, c / \sqrt{n}\right)$ and defining for short: $p_{k}^{n}=1-E\left[\mathbb{I}_{X_{k}^{(n)} \in A_{\Delta}\left(X_{i}, X_{j}\right)}\right]$, we have: $p_{k}^{n} \rightarrow 0$, for any $k$ when $n \rightarrow \infty$. Thanks to (6):

$$
\lim _{n \rightarrow \infty} \sum_{k=1}^{n} p_{k}^{n}<+\infty
$$

The assert is proved if we show that: $\lim _{n \rightarrow \infty} \prod_{k=1}^{n}\left(1-p_{k}^{n}\right)>$ $\epsilon>0$. By the continuity of $\log$ function, this is equivalent to:

$$
\lim _{n \rightarrow \infty} \sum_{k=1}^{n} \log \left(1-p_{k}^{n}\right)>\log \epsilon>-\infty
$$

Observe that $\log (1-x)>-2 x$ for any $0 \leq x \leq x_{0}$, with $x_{0} \approx 0.8$ such that $1-x_{0}=\exp \left(-2 x_{0}\right)$, and that for $n$ sufficiently large we can assume $p_{i}^{n}<x_{0}$; thanks to (11) we can easily show (12).

At last, to prove that $S^{*}$ selects w.h.p. transmission ranges which are $O(1 / \sqrt{n})$ it is sufficient to show that $\lim _{n \rightarrow \infty} \prod_{k \neq i, j} E\left[\mathbb{I}_{X_{k}^{(n)}(t) \in A_{\Delta}\left(X_{i}, X_{j}\right)}\right]=0$ whenever $d_{i j}^{(n)}=$ $\omega\left(\frac{1}{\sqrt{n}}\right)$. Also in this case, defining for short: $p_{k}^{n}=1-$ $E\left[\mathbb{I}_{X_{k}^{(n)} \in A_{\Delta}\left(X_{i}, X_{j}\right)}\right]$ it results: $\lim _{n \rightarrow \infty} \sum_{k=1}^{n} p_{k}^{n}=+\infty$. Since $\log (1-x) \leq-x$ :

$$
\lim _{n \rightarrow \infty} \sum_{k=1}^{n} \log \left(1-p_{k}^{n}\right) \geq-\sum_{k=1}^{n} p_{k}^{n}=-\infty
$$

and thus by continuity of exponential function: $\lim _{n \rightarrow \infty} \prod_{k=1}^{n}\left(1-p_{k}^{n}\right)=0$. 